\section{Korean Guidelines for Cardiopulmonary Resuscitation. Part 6. Pediatric basic life support}

\author{
Jisook Lee', Do Kyun Kim², Eun Kyeong Kang ${ }^{3}$, Jin-Tae Kim²,
} Jae Yoon $\mathrm{Na}^{5}$, Bobae Park ${ }^{6}$, Seok Ran Yeom ${ }^{7}$, Joo Suk $\mathrm{Oh}^{8}$, Won Kyoung Jhang ${ }^{9}$, Soo In Jeong ${ }^{10}$, Jin Hee Jung ${ }^{11}$, Yu Hyeon Choi ${ }^{12}$, Jea Yeon Choi ${ }^{13}$, June Dong Park ${ }^{12}$, Sung Oh Hwang ${ }^{14}$; on behalf of the Steering Committee of 2020 Korean Guidelines for Cardiopulmonary Resuscitation and Emergency Cardiovascular Care

\footnotetext{
'Department of Emergency Medicine, Ajou University School of Medicine, Suwon, Korea ${ }^{2}$ Department of Emergency Medicine, Seoul National University Hospital, Seoul, Korea

${ }^{3}$ Department of Pediatrics, Dongguk University Ilsan Hospital, Goyang, Korea

${ }^{4}$ Department of Anesthesiology and Pain Medicine, Seoul National University College of Medicine, Seoul, Korea ${ }^{5}$ Department of Pediatrics, Hanyang University Medical Center, Hanyang University College of Medicine, Seoul, Korea

${ }^{6}$ Department of Nursing, Seoul National University Hospital, Seoul, Korea

${ }^{7}$ Department of Emergency Medicine, Pusan National University College of Medicine, Busan, Korea

${ }^{8}$ Department of Emergency Medicine, The Catholic University of Korea College of Medicine, Seoul, Korea

${ }^{9}$ Department of Pediatrics, Children's Hospital Asan Medical Center, Seoul, Korea

${ }^{10}$ Department of Pediatrics, Ajou University Hospital, Suwon, Korea

${ }^{11}$ Department of Emergency Medicine, SMG-SNU Boramae Medical Center, Seoul, Korea

${ }^{12}$ Department of Pediatrics, Seoul National University College of Medicine, Seoul, Korea

${ }^{13}$ Department of Emergency Medicine, Gachon University Gil Medical Center, Gachon University College of Medicine, Incheon, Korea

${ }^{14}$ Department of Emergency Medicine, Yonsei University Wonju College of Medicine, Wonju, Korea
}

The 2020 Korean pediatric cardiopulmonary resuscitation (CPR) guideline is the medical recommendation derived on the basis of scientific evidence for pediatric basic life support (PBLS). This is based on the scientific consensus and treatment recommendations provided by the International Liaison Committee on Resuscitation in 2020, which provides the CPR guideline. Studies published for PBLS were additionally considered. ${ }^{1}$ The evidence of revised items that are of high clinical importance and require further consideration were reviewed in an acceptable adaptation or hybrid format and meta-analysis or scoping review was used.

\section{MAJOR CHANGES IN 2020 PBLS GUIDELINE}

Compared with the 2015 guideline, ${ }^{2}$ the changes in the 2020 PBLS guideline are as follows.

Endotracheal intubation or supraglottic airway (SGA) insertion versus bag mask ventilation (BMV)

The use of BMV is recommended rather than endotracheal intubation or SGA insertion for pediatric out-of-hospital cardiac arrest (OHCA) (Class Illb, Level C-LD). For pediatric in-hospital cardi-
elSSN: 2383-4625

Received: 7 March 2021

Revised: 3 April 2021

Accepted: 6 April 2021

Correspondence to: June Dong Park Department of Pediatrics, Seoul National University College of Medicine, 101 Daehak-ro, Jongno-gu, Seoul 03080, Korea

E-mail:jdparkmd@snu.ac.kr ORCID

https://orcid.org/0000-0001-8113-1384

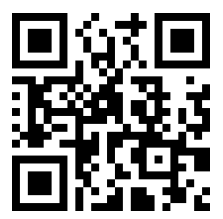

How to cite this article:

Lee J, Kim DK, Kang EK, Kim JT, Na JY, Park B, Yeom SR, Oh JS, Jhang WK, Jeong SI, Jung $J \mathrm{H}$, Choi YH, Choi JY, Park JD, Hwang SO; on behalf of the Steering Committee of 2020 Korean Guidelines for Cardiopulmonary Resuscitation and Emergency Cardiovascular Care. 2020 Korean Guidelines for Cardiopulmonary Resuscitation. Part 6. Pediatric basic life support. Clin Exp Emerg Med 2021;8(S):S65-S80. https://doi. org/10.15441/ceem.21.026

This is an Open Access article distributed under the terms of the Creative Commons Attribution Non-Commercial License (https:// creativecommons.org/licenses/by-nc/4.0/). 
ac arrest, there is no comparable evidence for applying endotracheal intubation or SGA insertion and BMV to date.

\section{Dispatcher-assisted CPR}

It is recommended to provide dispatcher-assisted CPR with the help of emergency services for pediatric OHCA (Class I, Level C-LD). If bystander CPR is not performed for OHCA, it is recommended to provide dispatcher-assisted CPR using the help of emergency services (Class I, Level C-LD). The recommendation to provide dispatcher-assisted CPR if the bystander CPR is performed for pediatric OHCA cannot be set with the evidence available to date.

\section{Compression-only CPR}

The rescuer should perform standard CPR with ventilation for infant and pediatric OHCA (Class I, Level B-NR). Although the bystander is unable to perform rescue breaths or has not been trained in it for pediatric OHCA, at least compression-only CPR should be done (Class I, Level B-NR).

\section{Chest compression based on end-tidal carbon dioxide $\left(\mathrm{ETCO}_{2}\right)$ monitoring}

It is not necessary to adjust the chest compression as per the measurements of $\mathrm{ETCO}_{2}$. However, it can be helpful to measure the $\mathrm{ETCO}_{2}$ if possible (Class Illb, Level C-LD).

\section{SEQUENCE OF PBLS}

\section{Pediatric chain of survival}

Basic life support (BLS) in pediatric cardiac arrest has a considerable impact on the return of spontaneous circulation (ROSC) and survival rate; it begins with the prevention of cardiac arrest, which is the first step in the chain of survival. The pediatric chain of survival begins with the recognition of cardiac arrest and a rescue request. However, before this, the prevention of cardiac arrest begins with several institutional strategies for injury prevention and safety outside the hospital. In the hospital, it is important to make efforts to prevent cardiac arrest using an early warning system. The first three among the five elements in the survival chain belong to BLS (Fig. 1). Similar to that in adults, for children, providing rapid and effective CPR by laypersons is helpful to regain successful ROSC and neurological recovery. For children, the survival rate considerably varies depending on age and cause of cardiac arrest, and it increases with age. For pediatric in-hospital cardiac arrest (IHCA), the survival rate is as high as $>40 \%{ }^{3}$

\section{Importance of prevention in pediatric cardiac arrest}

Cardiac arrest is difficult to predict and can occur in various places. Both non-medical factors such as cardiac arrest awareness and CPR education and medical factors such as prevention, treatment, and rehabilitation of cardiac arrest are all related to survival. The survival rate in cardiac arrest is low even with effective CPR; therefore, taking necessary precautions is important to prevent death from cardiac arrest. Respiratory failure and sudden infant death syndrome are the primary causes of infantile cardiac arrest. However, for children aged $>1$ year, trauma is the most common cause of cardiac arrest. Therefore, unlike that in adults, pediatric cardiac arrest can be prevented by appropriate management of the environment and changes in lifestyle. Sudden infant death syndrome can be prevented by not letting the child sleep on the stomach, not laying on a soft floor, and the caregiver's smoking cessation. Traffic accidents, which are the primary cause of injury, can be prevented by wearing a seat belt and installing a child car seat. Child safety seats vary by age. Baby safety seats facing the rear should be used for infants aged $<1$ year and weighing $<9 \mathrm{~kg}$; child safety seats should be used for children aged 1 to 4 years, and a secondary chair with a seat belt is necessary for children aged 4 to 7 years. If children aged $<12$ years sit in the front seat, airbag-related fatal injuries can occur, and the risk from improper seat belting increases. Drowning is the second most frequent cause of accidental death in children aged $<5$ years as well as the third most frequent cause of accidental death in adolescents. ${ }^{2}$ Most children drown in

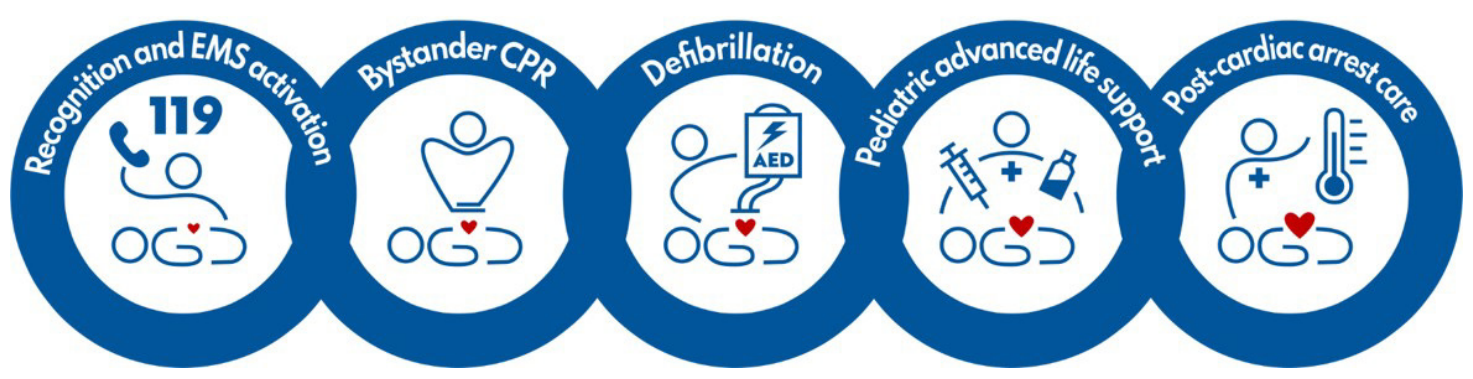

Fig. 1. Pediatric out-of-hospital chain of survival. EMS, emergency medical system; CPR, cardiopulmonary resuscitation; AED, automated external defibrillator. 
swimming pools while their caregivers are away. Adolescents often drown in either lake or river. Drowning can be prevented by wearing lifesaving gear during swimming. Recent domestic statistics show that murder by assault is the third cause of death among children aged between 1 and 9 years. Efforts to prevent such accidents should be made by attention and active reporting of child abuse. In adolescents aged > 10 years, the first cause of death is deliberate self-harm (suicide). It can be prevented by providing emotional support to adolescents and active interventions against suicide risk. ${ }^{4}$

\section{Recognition of cardiac arrest, rescue request, and bystander CPR}

Because an asphyxial cardiac arrest is more common in children, when a cardiac arrest is noticed, prompt CPR is as important as a rapid rescue request; however, because mobile phones are easily accessible in Korea, immediate rescue request should be made first, similar to that in adults. A domestic study on pediatric OHCA shows that the faster the CPR is performed after cardiac arrest, the higher the ROSC is, and rapid and effective layperson's CPR at the scene improves the rate of ROSC in pediatric OHCA. ${ }^{5}$ Furthermore, the neurological results are better when patients are discharged.

\section{PBLS FOR LAY RESCUERS}

There is a difference in the cause of cardiac arrest between children and adults. Moreover, because their sizes are different, CPR methods differ. However, it is difficult to distinguish between children and adults based on only one feature, and there is a lack of scientific evidence to determine the age at which different CPR methods should be applied. In this guideline, age was classified based on the applicability at the scene of cardiac arrest and educational ease. When a child is too big in size to be differentiated

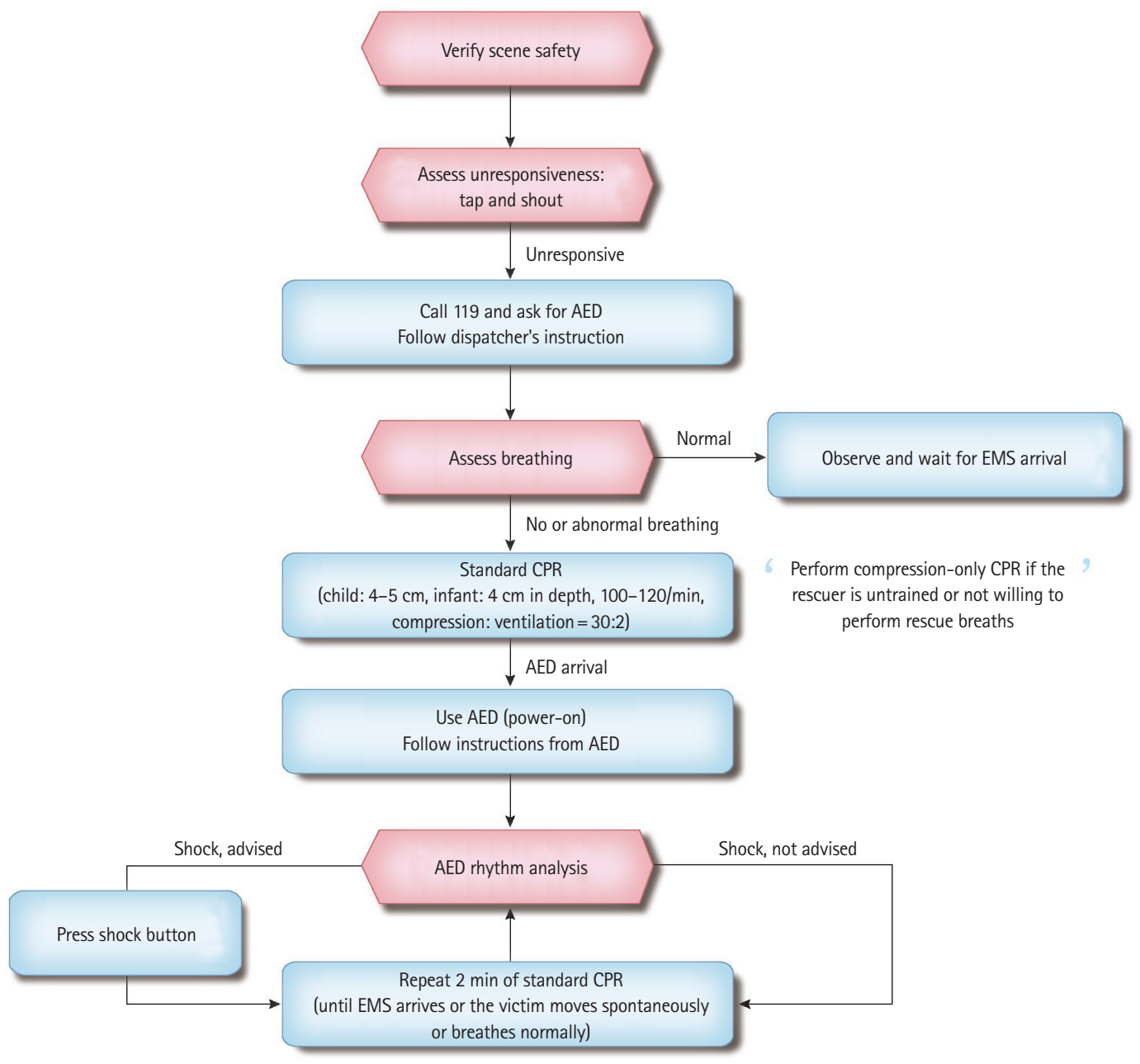

Fig. 2. Basic life support algorithm for pediatric out-of-hospital cardiac arrest (for lay rescuer). AED, automated external defibrillator; EMS, emergency medical system; CPR, cardiopulmonary resuscitation. 
from an adult, it is the discretion of the rescuer to determine which method, pediatric or adult CPR, can be applied. The rescuer can apply either method because the miscalculation of the age of the patient does not cause significant harm to the cardiac arrest patient. The definition of age in CPR is listed as follows: neonate, up to 4 weeks from birth; infant, $<1$ year old; pediatrics, from 1 to $<8$ years old; and adult, $\geq 8$ years old.

PBLS is applied to infants and children aged $<8$ years regardless of the layperson or the healthcare provider. Fig. 2 shows the algorithm for PBLS (Table 1). As shown in Fig. 3, for transmission risk of infectious diseases, such as severe acute respiratory syndrome and coronavirus disease-19 (COVID-19), compression-only CPR is performed rather than standard CPR. If rescuers perform CPR on an infant or child with infectious disease, they should wash their hands with soap and water or disinfect with alcoholbased hand sanitizer as soon as possible as per infection control rules and then change clothes. Moreover, they should contact local health authorities to verify for COVID-19 tests and then selfquarantine. Ventilation is extremely important in pediatric and infant CPR because asphyxial cardiac arrest is much more common than cardiac arrest. However, similar to that for adults, for infants and children with cardiac arrest, the order of CPR is to compress the chest first, followed by rescue breathing. It is to unify the education and training and also to continue the previous 2015 CPR guidelines. . $^{6}$

\section{Safety of rescuer and patient}

$A$ rescuer should verify the safety in the current area whenever CPR is performed. Theoretically, CPR has a risk of transmission of infectious diseases. Although the risk of rescuers being infected is very low, you must wear a mask and pay attention to personal protection during the COVID-19 pandemic.

\section{Recognition of cardiac arrest and response check}

First, check whether the patient needs CPR. If an unconscious patient is gasping or not breathing, you should determine that it is in cardiac arrest and requires CPR. Lightly tap the patient and shout, "Are you okay, kid?" If you know the name of that kid, call his/her name. Then, you should quickly check whether the child is injured and what medical treatment is required.

\section{Activation of the emergency medical system (EMS)}

If the patient does not respond to stimulation and no one else is around, shout for help. If there are people around you, ask them to call 119 and bring an automated external defibrillator (AED). If no one is nearby, the first discoverer immediately calls 119 for rescue. Considering the high penetration rate in Korea, most rescuers have a cell phone. Therefore, the rescuer should stay on the scene and make the phone call right away. Inform the dispatcher regarding the state of the unconscious patient, request an AED, and perform the subsequent steps as per the instructions of the emergency-dispatcher. If the patient is unresponsive and there are two or more rescuers, the first rescuer immediately starts $\mathrm{CPR}$, and the other rescuer activates the EMS while preparing for AED. If the rescuer is alone and does not have a cell phone, CPR must be first performed for 2 minutes, then EMS must be called, and nearby AED must be brought. The rescuer should return to the patient as soon as possible and use AED. If there is no AED, CPR with chest compression should be resumed.

\section{Verify the patient's breathing}

Remove the patient's upper garment, expose his/her chest, and verify the breathing. If the patient is regularly breathing, he does not require CPR. If there is no evidence of trauma, it can help maintain the airway and reduce the risk of aspiration to allow the patient to assume a recovery posture lying on his side. Repeatedly verify the patient's breathing status until emergency rescuers ar-

Table 1. Reference table of pediatric basic life support for lay rescuers

\begin{tabular}{ll}
\hline $\begin{array}{l}\text { Management } \\
\text { Breathing that requires CPR }\end{array}$ & If there is no breathing or only gasping \\
$\begin{array}{l}\text { Chest compression } \\
\text { Location: Sternum just below the line connecting the nipples for infants, the lower half of the sternum for children } \\
\text { Depth: At least one-third of the AP diameter of chest ( } 4 \mathrm{~cm} \text { for infants and } 4 \text { to } 5 \mathrm{~cm} \text { for children) } \\
\text { Rate: } 100-120 / \text { min } \\
\text { Chest compression-to-ventilation ratio }=30: 2\end{array}$ \\
$\begin{array}{l}\text { Chest compression to ventilation ratio } \\
\text { without advanced airway }\end{array}$ & $\begin{array}{l}\text { Perform compression-only CPR during COVID-19 pandemic } \\
\text { AED }\end{array}$ \\
AED analyzes rhythm & Turn on and use it as soon as you get AED \\
CPR after shock & Stop chest compression for rhythm analysis
\end{tabular}

CPR, cardiopulmonary resuscitation; AP, anteroposterior; COVID-19, coronavirus infectious disease 2019; AED, automated external defibrillator. 


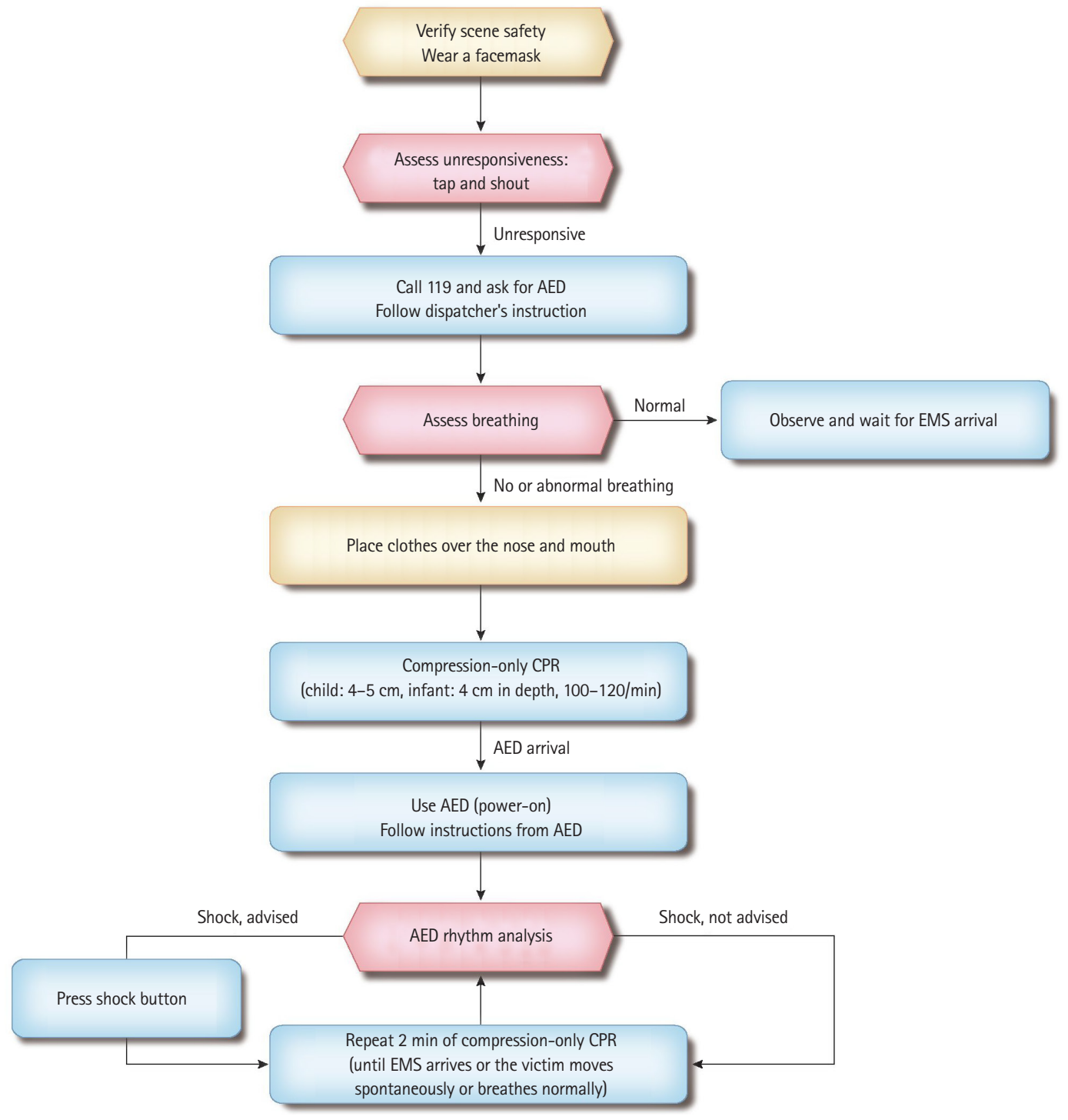

Fig. 3. Basic life support algorithm for pediatric out-of-hospital cardiac arrest (for lay rescuer during the COVID-19 pandemic). AED, automated external defibrillator; EMS, emergency medical system; CPR, cardiopulmonary resuscitation.

rive. Children with difficulty in breathing often take a posture where the airways will be more open and easier to breathe on their own. Therefore, if children with difficulty in breathing are attempting to take a more comfortable posture, let them maintain that posture. If the patient is unresponsive and is not breathing or is only gasping (cardiac arrest breathing), start CPR. If the patient's gasping breath is mistaken for normal breathing, CPR may be occasionally delayed. You should also consider a state of gasping breath as non-breathing and then start CPR.

\section{Chest compression}

Proper chest compression during cardiac arrest maintains blood flow to major organs and increases the possibility of ROSC. If the infant or child is not responding and is not breathing, immediately perform chest compression 30 times. The compression depth and rate do not change in the 2015 PBLS guideline. The appropriate compression rate is $100-120 / \mathrm{min}$, and the compression depth is one third of the anteroposterior diameter (chest thickness) at least or $4 \mathrm{~cm}$ depth in infants and $5 \mathrm{~cm}$ in children. ${ }^{9}$ It is best to perform chest compression on a flat, hard surface. There are limited studies on children using feedback devices for chest compressions. For infants, the lay rescuer and healthcare provider should compress the sternum just below the line connecting the nipples using two fingers when they perform CPR alone. At this time, be careful not to press on the xiphoid process and ribs. For children, you should compress the lower half of the sternum us- 
ing the heel of one or two hands. At this time, be careful not to press on the xiphoid process and ribs. Regardless of using one or two hands, minimum one third of the thoracic anteroposterior diameter $(4-5 \mathrm{~cm})$ should be maintained whenever compressing. It is important to ensure that the chest has completely recoiled to its normal position after each compression. Only when the chest completely recoils, the venous return to the heart can be sufficiently recirculated. Incomplete chest recoil is common during pediatric CPR, particularly when rescuers are exhausted. Incomplete chest recoil increases the pressure within the chest cavity and reduces venous return, coronary artery perfusion, cardiac output, and perfusion to cerebral arteries. Rescuer fatigue can make the speed, depth, and recoil of chest compressions inadequate. Even if the rescuer denies that he is exhausted and continues with $\mathrm{CPR}$, the quality of chest compression will deteriorate within minutes. If there are two or more rescuers, they should take turns performing chest compression every 2 minutes. This prevents the rescuer from getting tired and decreasing the quality and speed of chest compressions. Taking turns in chest compression should be performed as soon as possible (ideally within 5 seconds) to minimize its interruption. For pediatric cardiac arrest, the lay rescuer should perform CPR by repeating cycles of 30 chest compressions and two ventilations until the EMS specialists arrive or the patient can breathe on his own. To obtain good results, for the pediatric and infant CPR in whom asphyxial cardiac arrest is common, chest compressions and ventilation should be administered together. The person who performs pediatric inhospital and out-of-hospital CPR should perform conventional CPR with ventilation and chest compressions. If the rescuer is unwilling or untrained to perform rescue breathing or if there is a situation in which ventilation is not possible, he should perform at least compression-only CPR until the EMS specialist arrives.

\section{Airway opening and ventilation}

The ratio between chest compression and ventilation of one rescuer is 30:2. First, perform chest compression 30 times, open the airway, and then deliver ventilation two times. In infants or children who do not respond, the tongue may block the airway; therefore, regardless of trauma, open the airway by tilting the head and lifting the jaw.

Use either mouth-to-mouth or mouth-to-nose ventilation for infants or mouth-to-mouth ventilation for children. When breathing in, ensure that the chest rises and each breath takes 1 second. If the chest does not rise, verify the head position again to open the airway, close the mouth more tightly to prevent respiration leakage, and then attempt ventilation. Moreover, it is necessary to identify a position for optimal airway maintenance and effec- tive ventilation by adjusting the degree of head tilt. If it is difficult to breathe via the mouth and nose at the same time when giving breath to infants, mouth-to-mouth or mouth-to-nose ventilation can be performed. For mouth-to-mouth ventilation, pinch the nose, whereas for mouth-to-nose ventilation, cover the mouth.

If there is only one rescuer, two ventilations after 30 chest compressions should be performed for as short a time as possible to minimize chest compression interruption time. If there are two rescuers, one person is in charge of chest compressions and the other is in charge of ventilation. After 30 chest compressions, sequentially perform ventilation twice. You should then make efforts to minimize chest compression interruption by ensuring that both chest compressions and ventilation are continuously performed.

\section{Chest compression to ventilation ratio}

After two ventilations, immediately perform 30 chest compressions. It takes approximately 2 minutes for one rescuer to perform five cycles of chest compressions and ventilations with a ratio of 30:2. If there are two rescuers, they should perform chest compressions and ventilation by turns after five cycles.

\section{Compression-only CPR}

In the observational studies published since 2015 for infants and children, conventional CPR with ventilation showed a better overall prognosis than chest compression-only CPR without ventilation. ${ }^{10-14}$ However, for infants, certain studies show a poor overall outcome regardless of the CPR methods. There have been reports that chest compression-only CPR showed outcomes similar to those of conventional CPR in children aged $>8$ years. For children, the prognosis was better when compression-only CPR was performed than not performing CPR at all. For infants, similarly, prognosis was poor when compression-only CPR was performed than not performing CPR at all, and conventional CPR with ventilation was associated with a good prognosis. Overall, conventional CPR with ventilation should be performed for infants and children. However, if the rescuer is unable or unwilling to perform ventilation, he should start compression-only CPR first; after that if possible, perform conventional CPR with ventilation.

\section{Dispatcher-assisted CPR}

A layperson who first witnesses a cardiac arrest may experience delays and difficulties in confirming cardiac arrest and performing CPR because he is not educated about it. Emergency service consultants can help the layperson to verify the cardiac arrest and perform CPR via telephone guidance. If a dispatcher instructs CPR over the phone for a pediatric OHCA patient, the possibility 
of performing CPR by rescuer increases and the time required to start CPR decreases..$^{15-17}$ Therefore, it is recommended to provide telephone-assisted CPR for pediatric OHCA. If the bystander is not undergoing CPR, the emergency consultant should be trained to help start CPR.

\section{PBLS FOR HEALTHCARE PROVIDER}

PBLS for healthcare provider has certain differences from for the lay rescuer; however, they are similar (Table 2 and Figs. 4, 5). 18,19 Healthcare providers are mostly working in teams; therefore, each process is often simultaneously performed (chest compression and preparation of ventilation). Therefore, the priority of the activity is relatively less emphasized.

\section{Safety of rescuer and patient}

A rescuer should verify the safety in the current area whenever CPR is performed. Healthcare providers during the COVID-19 pandemic are recommended to use personal protective equipment (PPE) while performing aerosol-generating procedures in CPR. It is recommended that healthcare providers wear appropriate PPE, including masks, gloves, goggles, and surgical gowns, because chest compression and ventilation can cause the formation of aerosols and increase the risk of transmission of infection. When defibrillation is strongly recommended, it is suggested to actively implement it while paying attention to the transmission of infection (Fig. 6). Although compression-only CPR has a low risk of transmission of infection, it is suggested to cover the patient's mouth if possible because there is a potential for aerosol generation. The rescuer should wash hands with soap and water or disinfect his hands with alcohol-based hand sani- tizer as soon as possible as per infection control rules, and then change his clothes after finishing CPR. Moreover, the rescuer should contact local health authorities to verify for COVID-19 tests and self-isolation.

\section{Check the response}

First, verify whether the patient requires CPR. Tap the patient lightly and shout, "Are you okay, kid?" If you know the name of that kid, call his name. Quickly verify whether the child is injured and what medical treatment is required.

\section{Activation of the EMS}

If the patient is unresponsive and does not breathe (or gasping/ abnormally breathing), ask someone nearby to call 119 and bring an AED. In the hospital, a witness immediately asks for help, ask a nearby person to bring a defibrillator, verify the pulse and breathing of the patient within 10 seconds, and start CPR if there is no pulse or breath.

\section{Check the pulse}

If the infant or child has no response and is not breathing normally, the care provider should check his pulse. The pulse check time should not exceed 10 seconds. The pulse is verified in the brachial artery in infants and the carotid or femoral artery in children. If you cannot feel the patient's pulse within 10 seconds or are unclear if there is a pulse, start chest compression. ${ }^{20}$

\section{Insufficient breathing and adequate pulse with good perfusion}

If the patient's pulse is $>60 / \mathrm{min}$ but breathing is inappropriate, provide rescue breathing at a rate of $12-20 /$ min until spontaneous breathing is recovered (breathing once every 3 to 5 seconds).

Table 2. Reference table of pediatric basic life support for healthcare providers

\begin{tabular}{|c|c|}
\hline Management & Content \\
\hline Breathing that requires CPR & If there is no breathing or only gasping \\
\hline Check pulse and breathing & Check pulse and look for no breathing or abnormal breathing simultaneously within 10 seconds \\
\hline Chest compression method & $\begin{array}{l}\text { One rescuer for infant: two-finger chest compression } \\
\text { Two or more rescuers for infant: two-thumb encircling chest compression } \\
\text { Children: heel of one or two hands chest compression }\end{array}$ \\
\hline Chest compression & $\begin{array}{l}\text { Location: sternum just below the line connecting the nipples for infants, the lower half of the sternum for children } \\
\text { Depth: at least one-third of the AP diameter of chest ( } 4 \mathrm{~cm} \text { for infants and } 4 \text { to } 5 \mathrm{~cm} \text { for children) } \\
\text { Rate: } 100-120 / \mathrm{min}\end{array}$ \\
\hline Chest compression to ventilation ratio & $\begin{array}{l}\text { (One rescuer) chest compression: ventilation }=30: 2 \\
\text { (Two or more rescuers) chest compression: ventilation }=15: 2\end{array}$ \\
\hline Ventilation after return of spontaneous circulation & $12-20 / \mathrm{min}$ (once every 3 to 5 seconds) if the pulse rate is $>60 / \mathrm{min}$ and the perfusion condition is good \\
\hline After insertion of advanced airway & 10 ventilation/min (once every 6 seconds) regardless of chest compression \\
\hline Rhythm analysis & Stop chest compression for rhythm analysis \\
\hline CPR after defibrillation & Resume chest compression immediately after defibrillation \\
\hline
\end{tabular}

$\mathrm{CPR}$, cardiopulmonary resuscitation; $\mathrm{AP}$, anteroposterior. 


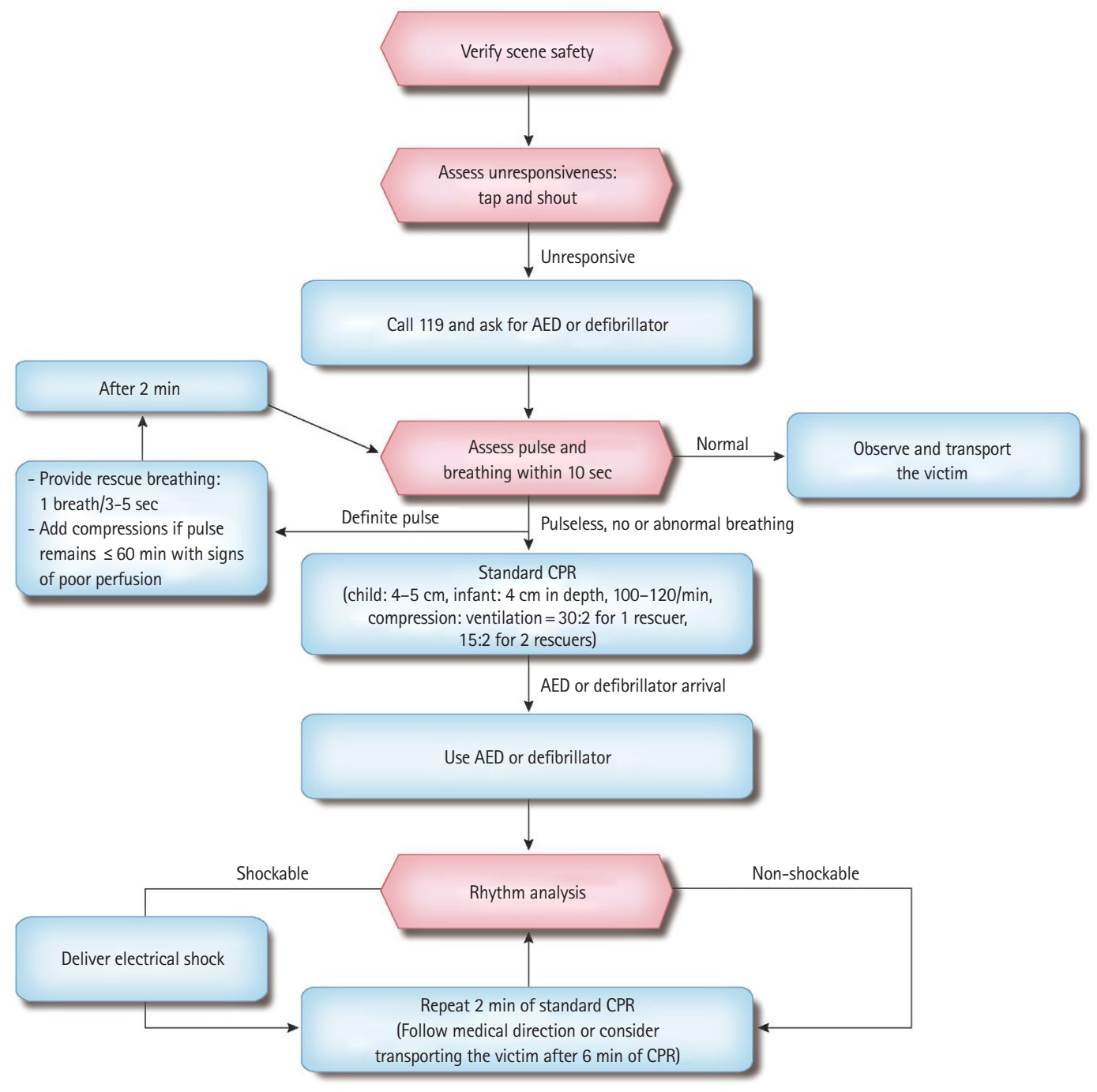

Fig. 4. Basic life support algorithm for pediatric out-of-hospital cardiac arrest (for healthcare provider). AED, automated external defibrillator; CPR, cardiopulmonary resuscitation.

The pulse is then rechecked every 2 minutes and the pulse checking time should not exceed 10 seconds.

\section{Bradycardia with poor perfusion}

If the patient's pulse is $<60 / \mathrm{min}$ and the perfusion is poor even with oxygen and ventilation (i.e., the skin is pale, mottled, or cyanotic), start chest compression. Slow pulse with poor perfusion is a sign of the requirement for chest compression in infants and children because cardiac output in them largely depends on the heart rate (HR). CPR should be immediately performed before the occurrence of cardiac arrest to improve survival rate. The absolute criterion for HR at which chest compression should be started is not yet clear. It is recommended to perform chest compression when HR is $<60 / \mathrm{min}$ and the perfusion is not good for the convenience of education and memory of skills.

\section{Chest compression}

If the infant or child is not responding and there is no breathing and pulse (or if it is unclear whether there is a pulse), start chest compression. The difference in method used by the healthcare provider and that by the lay rescuer lies in how to compress the chest in infants. When the healthcare provider is alone, two-finger chest compression is used for infants. When there are two or more rescuers, two-thumb encircling chest compression is performed. It is to stretch the hand and wrap the infant's rib cage with both hands and strongly press the compression point of the sternum with both thumbs side-by-side.

The advantage of the two-thumb encircling chest compression is that it increases the coronary artery perfusion pressure more than that by the two-finger chest compression. It can consistently maintain adequate compression depth and force and can pro- 


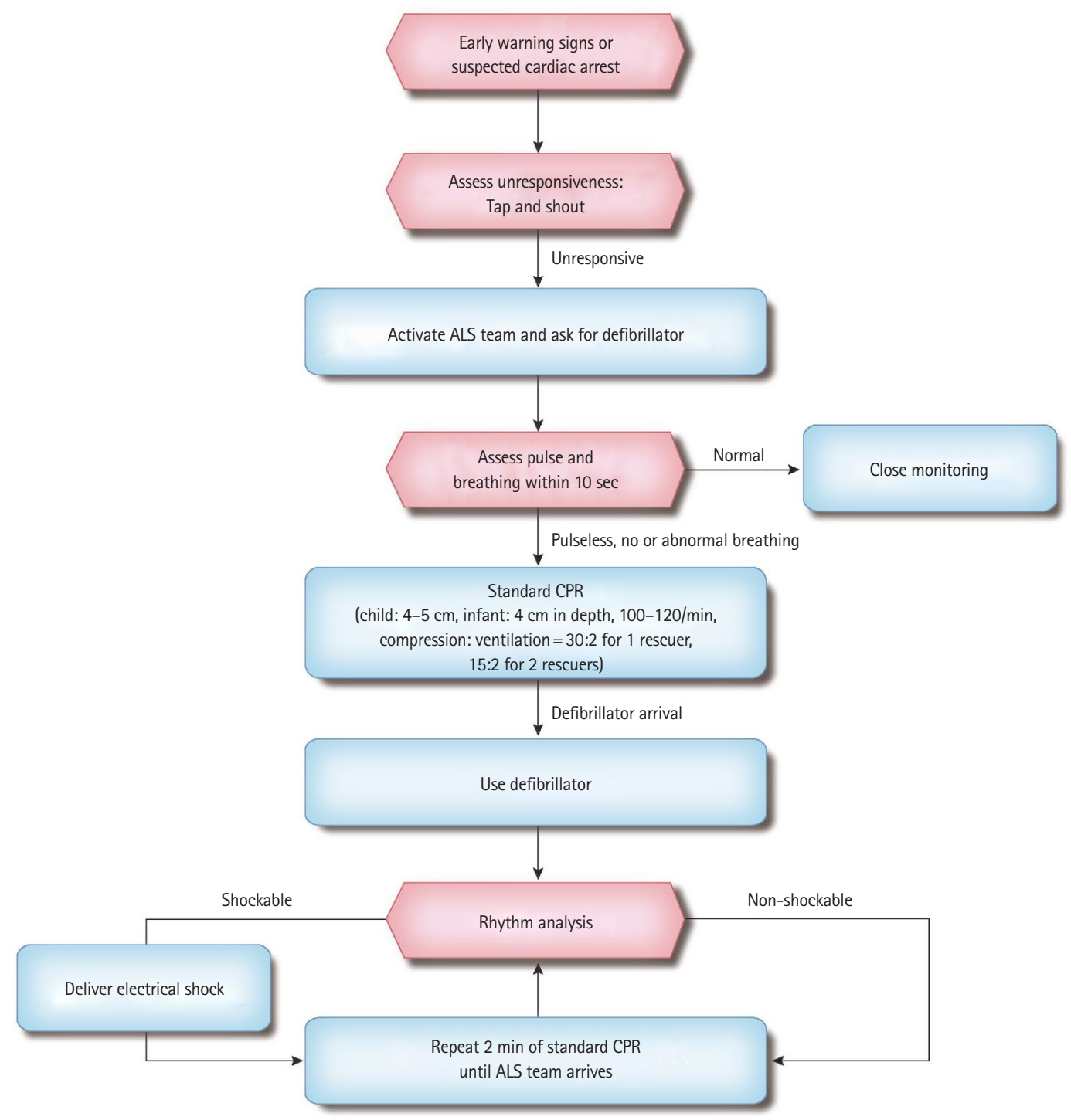

Fig. 5. Basic life support algorithm for pediatric in-hospital cardiac arrest (for healthcare provider). CPR, cardiopulmonary resuscitation; ALS, advanced life support.

duce higher systolic and diastolic pressures. If you cannot hold the patient's rib cage with both hands, just press the chest with two fingers.

The location of chest compression is the sternum right below the line connecting the nipples in infants and the lower half of the sternum in children. For one rescuer, the ratio of chest compression-to-ventilation is 30:2 using the two-finger chest compression. For two or more rescuers, the ratio of chest compression-to-ventilation is 15:2 using two-thumb encircling chest compression.

\section{Airway opening and ventilation}

Open the airway by tilting the head and lifting the jaw, and perform rescue breathing twice after 30 chest compressions (15 com- pressions if there are two rescuers). If there are signs of trauma that suggest spinal damage, open the airway with a jaw lift rather than a head tilt. It is very important to open the airway and perform ventilation in pediatric resuscitation; therefore, if you cannot open the patient's airway with only jaw-lifting, you should apply the head tilting regardless of the trauma.

If it is difficult to breathe through the mouth and nose at the same time when giving ventilation to infants, mouth-to-mouth or mouth-to-nose ventilation can be performed. For mouth-tomouth ventilation, make the patient breathe through the mouth while pinching the patient's nose. Confirm whether the patient's chest rises when inhaling from both sides. 


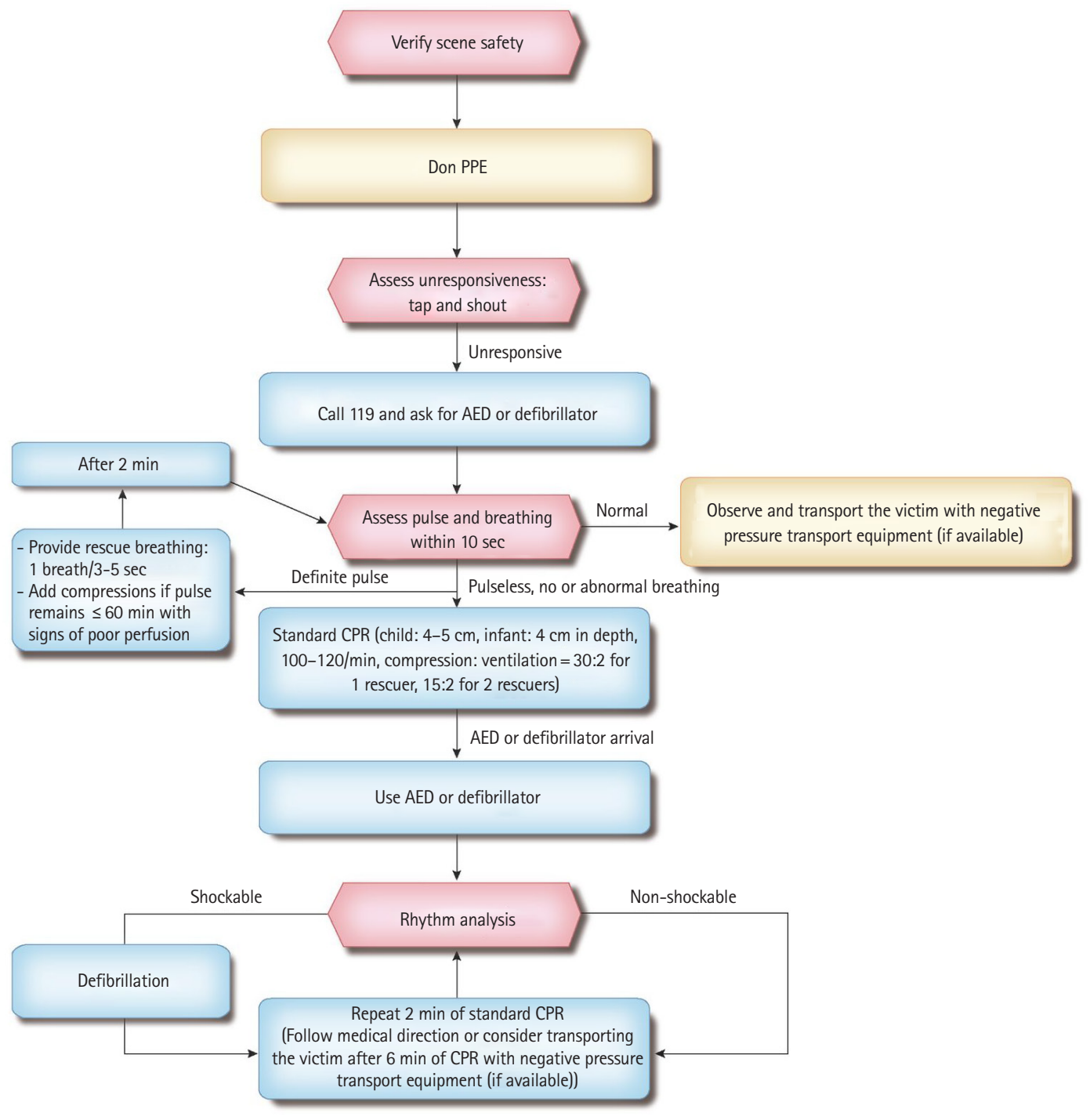

Fig. 6. Basic life support algorithm for pediatric out-of-hospital cardiac arrest (for healthcare provider during COVID-19 pandemic). PPE, personal protective equipment; $A E D$, automated external defibrillator; $C P R$, cardiopulmonary resuscitation.

\section{Equipment and methods related to ventilation Protective equipment}

As some rescuers are reluctant about the direct contact by mouthto-mouth ventilation, they should use PPE. The use of PPE is not $100 \%$ effective in preventing the spread of infection and can create resistance to airflow. Therefore, ventilation should not be delayed in using PPE.

\section{Bag-mask ventilation (BMV)}

Most pediatric cardiac arrest patients can have adequate ventilation with bag mask breathing; however, it has the disadvantage of frequent interruption of chest compressions and a high risk of airway aspiration compared with the insertion of an advanced airway (endotracheal intubation or SGA). But, it requires more time to master the intubation or SGA skills than to master BMV. In pediatric $\mathrm{OHCA}$, intubation failure rates and complications are higher than those in the BMV. From these points, it is reasonable to apply the BMV rather than intubation or SGA for rescue breathing of pediatric OHCA patients. It is important to learn techniques such as selecting the appropriate size for the mask, opening the airway, fitting the mask close to the face, and applying appropriate pressure for effective BMV.

\section{Ventilation bag}

Pediatric self-inflating bags supply at least 450 to $500 \mathrm{~mL}$ air, and the bags with a smaller capacity may not provide sufficient tidal volume for term infants. For older children or adolescents, an adult self-inflating bag (approximately 1,000 $\mathrm{mL}$ ) should be 
used. If oxygen is not supplied, it is ventilated only with room air; if the amount of oxygen supplied is $10 \mathrm{~L} / \mathrm{min}$, the oxygen concentration is maintained from $30 \%$ to $80 \%$. To supply a higher concentration (60\%-95\%) of oxygen, connect an oxygen reservoir to the bag. $0 x y g e n$ at $10-15 \mathrm{~L} / \mathrm{min}$ can be supplied to the reservoir attached to the pediatric bag and at least $15 \mathrm{~L} / \mathrm{min}$ to the adult bag.

\section{Ventilation method to prevent hyperventilation}

Hyperventilation reduces circulating blood flow; therefore, it is important to prevent it. Two ventilations are performed after 30 chest compressions (by one rescuer) or 15 chest compressions (by two healthcare providers) before using advanced airway (insertion of an endotracheal tube or SGA devices is achieved); at this time, mouth to mouth ventilation or BMV is used. After performing advanced airway management, the "compression to ventilation ratio" of CPR is not used. Continue chest compressions at a rate of 100-120/min without interruption and ventilation 10 times per minute. Two or more healthcare providers should rotate the compression role every 2 minutes to prevent exhaustion. If the patient has ROSC but has no breathing, perform only ventilation 12-20 times/min (once per 3-5 seconds). Excessive ventilation during CPR decreases venous return, thereby reducing cardiac output and cerebral blood flow and reducing coronary artery perfusion because of an increase in intrathoracic pressure. Therefore, the rescuer must ventilate according to the number per minute. Hand-compressed bags can generate high pressure; therefore, ventilate only enough to observe the chest rise.

\section{Two rescuers $B M V$}

BMV together by two rescuers helps with effective breathing when there is severe airway obstruction and poor lung elasticity or when it is difficult to fit the mask to the face tightly. One rescuer maintains the airway open with both hands and attaches the mask firmly to the patient's face and the other rescuer squeezes the ventilation bag. Two rescuers should verify whether the patient's chest is rising.

\section{Gastric distention and applying cricoid pressure}

You should avoid gastric distention because it impairs efficient ventilation and may cause vomiting. To minimize gastric distension, every breath can be performed over a second to avoid overpressure during inhalation.

Applying cricoid pressure may be considered to prevent air from entering the stomach. However, it is not recommended to routinely perform cricoid pressure. Cricoid pressure should be considered only when the patient is unconscious and there is another healthcare provider. Moreover, be aware that excessive pressure on the cricoid cartilage can block the trachea.

Oxygen

Animal experiments show that 100\% oxygen is harmful, but human studies show no detrimental effects of oxygen concentration after the neonatal period; therefore, supply 100\% oxygen during resuscitation. When the patient is stabilized, supply oxygen while verifying the oxygen concentration. The administration of humidified oxygen can prevent dryness of the mucous membranes and thickening of lung secretions.

Oxygen is administered using a mask or nasal cannula depending on the patient's breathing condition. The mask supplies 30\% to $50 \%$ oxygen when spontaneous breathing occurs. High concentrations of oxygen can be administered by supplying $15 \mathrm{~L} / \mathrm{min}$ of oxygen with a mask that tightly fits on the face and has a reservoir. When there is spontaneous breathing, use a nasal cannula suitable for the size of infants and children. When using a nasal cannula, up to 0.5-1 L/min can be administered for neonates, 1-2 L/min for infants, $4 \mathrm{~L} / \mathrm{min}$ for preschool children, and $6 \mathrm{~L} / \mathrm{min}$ for school-age children. The oxygen concentration is adjusted as per the child's size, respiratory rate, and breathing effort. When $2 \mathrm{~L}$ min of oxygen is administered to an infant, the inhaled oxygen concentration is $55 \% .^{21}$

\section{Defibrillation}

Ventricular fibrillation (VF) and pulseless ventricular tachycardia (pVT) may be the cause of sudden cardiac arrest or occur during CPR. A sudden collapse in children with witnesses (e.g., collapse during exercise) is attributed to VF or pVT, requiring immediate CPR and rapid defibrillation. VF and pVF are classified as "shockable rhythm" because they respond to defibrillation.

For infants, it is beneficial to use a manual defibrillator if a welltrained healthcare provider has identified the shockable rhythm. The first energy dose is $2 \mathrm{~J} / \mathrm{kg}$ and the second one is $\geq 4 \mathrm{~J} / \mathrm{kg}$ during defibrillation, which should not exceed $10 \mathrm{~J} / \mathrm{kg}$ or the maximum dose for adults. ${ }^{22}$

If you do not have a manual defibrillator, use an AED with a pediatric impulse attenuator for infants. Use an AED with a pediatric energy attenuator or pediatric patches for children aged $<8$ years. However, if you have neither a manual defibrillator nor an AED with an energy attenuator, an AED for adults that cannot control the dose of energy can be used for infants.

Rescuers should minimize the interval time between chest compressions and defibrillation and resume CPR by immediately restarting chest compressions after one defibrillation. The AED encourages the rescuer to reanalyze the rhythm every 2 minutes, 
and the defibrillation should ideally be immediately performed after the chest compression.

\section{RESUSCITATION IN SPECIAL CIRCUMSTANCES}

\section{Airway obstruction (choking) by foreign bodies}

Note that $>90 \%$ of deaths by a foreign body aspiration occur in children aged $<5$ years, of which $65 \%$ occur in infants..$^{23}$ The clinical symptoms of airway obstruction by a foreign body include sudden shortness of breath, coughing, nausea, grunting, and wheezing. It suddenly occurs and has no previous fever or respiratory symptoms, which distinguishes it from other respiratory difficulties. Airway obstruction caused by foreign bodies can range from mild to severe. If airway obstruction is mild, the child may cough or make a sound. Symptoms suspected of complete obstruction include no speech, no sound when coughing, unable to breathe, cyanosis, or loss of consciousness; in this case, immediate treatment is required.

If it is determined that airway obstruction is severe in children aged $>1$ year, perform back blow until a foreign body comes out or consciousness disappears. ${ }^{24}$ For infants, perform the back blow five times and chest thrust five times until a foreign body comes out or the consciousness disappears repeatedly. ${ }^{25,26}$ Abdominal thrust is not performed in infants owing to the high risk of internal organ damage because the ribs do not sufficiently protect the epigastric organs and the liver is relatively large. ${ }^{25}$

If the patient does not respond or his reaction disappears during the foreign body removal procedure, start CPR regardless of the presence or absence of a pulse. ${ }^{27}$ However, if you can see a foreign body in the patient's mouth before breathing after chest compression, use your fingers to remove it. If you cannot see a foreign body in the patient's mouth, do not try to remove it using your fingers. This blind swabbing of the mouth may push the foreign body deeper into the pharynx or damage the pharynx. ${ }^{27,28}$ After two ventilations, perform chest compression and ventilation repeatedly until the foreign body is removed. In fact, there are limited high-quality studies that can help determine guidelines for airway obstruction by foreign bodies. Most airway obstruction caused by a foreign body is resolved by letting the patient cough himself. However, in severe cases, the patient may need help from a rescuer. ${ }^{29,30}$

\section{Trauma}

Unintended trauma can be a leading cause of death in children and adolescents. Cardiac arrest by major blunt and penetrating injuries in children has a very high mortality rate. ${ }^{31-33}$ Tension pneumothorax, hemothorax, lung injury, and cardiac tamponade can interfere with hemodynamics, oxygenation, and ventilation. Therefore, you should always suspect severe chest injury in patients with chest-abdominal trauma. Early treatment for reversible causes after cardiac arrest owing to penetrating trauma can increase survival. ${ }^{34,35} \mathrm{It}$ is recommended in the guidelines to control bleeding, recover circulating blood volume, secure airways, and treat tension pneumothorax for cardiac arrest caused by trauma. These procedures should be concurrently performed with standard CPR. The principles of basic CPR for injured children are the same as for children with common diseases; however, there is certain emphasis. Improperly performed CPR can increase preventable mortality. Errors in opening and maintaining the airways and errors caused by not recognizing internal bleeding are common in resuscitating children. The points to be noted when performing CPR in pediatric trauma patients are listed below. ${ }^{36}$ 1) Cardiac arrest in pediatric trauma patients often occurs because breathing is not adequately maintained owing to hypovolemia by trauma. Therefore, it must be determined whether the airway and ventilation are maintained even in trauma patients. 2) If there is a possibility of airway obstruction because of broken teeth or blood, use a suction device. 3) If there is external bleeding, press to stop it. You should remove the patient's clothes and check his entire body to determine the bleeding points. After verification, ensure to cover with a warm cloth such that hypothermia does not occur. 4) If there is a possibility that a spinal injury occurred judging by the mechanism of the injury, then minimize cervical spine movement and do not pull or move the head and neck. Open the airway by lifting the patient's jaw and do not tilt the head. If the airway is not maintained by lifting jaw, secure the airway by tilting the head and lifting the chin. If there are two rescuers, one opens the airway and the other prevents cervical spine movement. At least the thighs, pelvis, and shoulders are fixed together on the spinal board. Because infants and toddlers have relatively large heads, the occipital part should be placed in a slightly recessed position than the torso, or the torso should be laid in a slightly elevated position and fixed to the spinal correction board to prevent cervical flexure. ${ }^{37}$ 5) Transfer children with multiple organ trauma to a trauma center with a pediatric specialist if possible. 6) Open thoracotomy could be considered for children with no pulse with a penetrating injury. ${ }^{234,38-40}$ However, there is still insufficient evidence to recommend performing emergency thoracotomy in children and infants without a pulse because of blunt trauma. ${ }^{41,42}$

\section{Drowning}

Drowning time is an important predictor of prognosis. Age, promptness of first aid, water type (freshwater or seawater), water temperature, and presence of witnesses will not be reliable prognos- 
tic factors. ${ }^{42}$ For drowning in ice water, the rescue time can be extended because there is a possibility of survival even if the drowning time is long. ${ }^{43,44}$ After removing the drowned children from water, initiate CPR immediately. Rescuers with special training initiate rescue breathing while underwater. Do not perform chest compressions in water because they are ineffective. ${ }^{45}$

There is no evidence that water acts as a foreign body causing airway obstruction. Therefore, you do not waste time trying to drain water from the patient's lungs. ${ }^{46}$ Open the airway, perform rescue breathing twice, and start CPR with chest compressions. If there is one rescuer, perform chest compressions and ventilations five times cyclically at the ratio of 30:2, call the EMS, and ask for an AED. If there are two rescuers, the first rescuer continues CPR, whereas the second rescuer reports to the EMS and prepares an AED. ${ }^{47}$

\section{Children who require special medical assistance}

Children who require special medical assistance because of complications owing to chronic disease (e.g., blockage of tracheostomy), problems with auxiliary medical devices (e.g., ventilator malfunction), and exacerbation of the existing disease need appropriate management for their conditions. If there is no information on an existing disease, treatment plans, and current medications, you may have difficulty performing treatments. Parents or caregivers should copy the child's medical information and place it at home, school, or care facility in advance. If the child is discharged from the hospital with a chronic or fatal illness, parent, school nurse, and home care provider should be aware of information about the reason for hospitalization, the condition during the hospitalization, and any symptoms that may worsen as well as receive training for $\mathrm{CPR}$ in special circumstances. ${ }^{48}$

\section{Children with prior medical directions}

If a decision has been made to limit or abandon CPR, medical personnel should specify CPR-related limitations in detailed prescribing instructions. The doctor should separately specify the prescribing instructions for situations outside the hospital. Care facilities and school nurses should copy and place the form of a child for whom decision of giving up on resuscitation attempts has been made. Parents, school nurses, and home-visit health care providers should receive sufficient information about the guide of CPR and the contact information in case of an emergency. ${ }^{49}$

\section{Ventilation through tracheostomy or tracheostomy window}

Caregivers of a child who received a tracheotomy (parent, school nurse, and home health care provider) should know how to maintain the child's airways, how to remove airway secretions, and how to perform CPR through artificial airways. They should perform assisted ventilation through a tracheostomy window and verify the airway maintenance of the child and whether his chest is rising. If ventilation through the tracheostomy window is not effective even with suction, it should be suspected that the tracheostomy tube was not properly inserted and airway maintenance should be checked again. When the patient's breathing is not appropriate, perform mouth to tracheostomy window ventilation for the patient who has had a tracheostomy. If the tracheostomy window is already blocked, perform BMV via the nose and mouth. ${ }^{50}$

\section{PREVENTION OF PEDIATRIC CARDIAC ARREST}

\section{Rapid response system for preventing pediatric cardiac arrest}

Rapid response systems have been employed in several medical institutions to prevent cardiac arrest by detecting physiological changes in hospitalized patients early and to reduce admission to the intensive care unit. ${ }^{51}$ This is to identify the clinical deterioration of the patient due to a delay of a call to medical staff in a hospital. It is an active effort to issue an early warning of changes in a patient's condition by scoring blood pressure, pulse, respiratory rate, body temperature, oxygen saturation, and level of consciousness. ${ }^{2}$

The use of a pediatric early warning score has reduced the incidence of cardiac arrest, length of stay in the hospital, and mortality as well as unplanned transfer to the pediatric intensive care unit. ${ }^{53,54}$ However, there are not many studies on the frequency of early response as per the working hours of the rapid response team (RRT) and the patient's prognosis, and there is currently no consensus on the standard for activating the domestic RRT; therefore, continuous further study is required. ${ }^{55}$

Moreover, when using pediatric early warning scores in medical institutions, it is necessary to discuss how to organize items to sensitively identify changes in the patient's condition. It is necessary to discuss whether to include only vital signs or also include sepsis, dyspnea, anaphylactic shock, hypovolemic shock, obstructive shock, arrhythmia, and metabolic acidosis. ${ }^{48}$ Moreover, it is necessary for the medical staff to quickly recognize and respond after discussing how to identify the false positive alerts of early warning score.

\section{Pediatric RRT (or medical emergency team)}

RRT is a department dedicated to first aid and monitoring of patients with deteriorating conditions in the hospital. It is composed 
of specialized medical staff to quickly identify and respond to signs that appear before a cardiac arrest occurs. Depending on the hospital's resources, the composition, operation method, and activation of the rapid response system can be adpated. Medical institutions form RRTs to better understand the patient's respiratory system, cardiovascular system, and changes in consciousness, and it comprises skilled medical staff for rapid intervention and efficient critical care management. ${ }^{56,57}$

There is not sufficient evidence that the operation of the pediatric RRT increases the rate of ROSC and decreases the mortality rate of pediatric cardiac arrest in hospitals. However, studies are showing that it reduces the incidence of pediatric cardiac arrest in hospitals, particularly outside the intensive care unit. ${ }^{58-61}$ In one cohort study, for children requiring unplanned admission to the pediatric intensive care unit, RRT implementation was associated with reduced mortality. ${ }^{62}$ Therefore, medical institutions with trained professional staff may consider establishing and operating a pediatric RRT to prevent the occurrence of cardiac arrest.

\section{CONFLICT OF INTEREST}

No potential conflict of interest relevant to this article was reported.

\section{ACKNOWLEDGMENTS}

This study was supported by a grant (2020E330300) of the Korean Disease Control and Prevention Agency funded by the Ministry of Health and Welfare, Republic of Korea.

We thank Ms. So Yeong Kim (EMT) for her assistance with administrative affairs and Mr. Myung Ha Kim for his assistance with literature searches for updating Korean guidelines for cardiopulmonary resuscitation. We also thank the Korean Association of Cardiopulmonary Resuscitation (KACPR) for supporting the process of proofreading.

\section{REFERENCES}

1. Maconochie IK, Aickin R, Hazinski MF, et al. Pediatric Life Support 2020 International Consensus on Cardiopulmonary Resuscitation and Emergency Cardiovascular Care Science With Treatment Recommendations. Pediatrics 2021;147(Suppl 1): e2020038505B.

2. Kim DK, Jhang WK, Ahn JY, et al. Part 6. Pediatric advanced life support: 2015 Korean Guidelines for Cardiopulmonary Resuscitation. Clin Exp Emerg Med 2016;3(Suppl):S48-61.

3. Nolan JP, Monsieurs KG, Bossaert $L$, et al. European Resusci- tation Council COVID-19 guidelines executive summary. Resuscitation 2020;153:45-55.

4. Statistics Korea. Statistics on the cause of death 2019 [Internet]. Daejeon: Korean Statistical Information Service; 2020 [cited 2020 Dec 19]. Available from: https://kosis.kr/statisticsList/statisticsListIndex.do?menuld = M_01_01Etvwcd = MT_ ZTITLEEtparmTabld = M_01_01\&toutLink= YctentrType = \#cont ent-group.

5. Park CB, Shin SD, Suh GJ, et al. Pediatric out-of-hospital cardiac arrest in Korea: a nationwide population-based study. Resuscitation 2010;81:512-7.

6. Fraser J, Hill C, McDonald D, Jones C, Petros A. The use of the laryngeal mask airway for inter-hospital transport of infants with type 3 laryngotracheo-oesophageal clefts. Intensive Care Med 1999;25:714-6.

7. Iohom G, Lyons B, Casey W. Airway management in a baby with femoral hypoplasia-unusual facies syndrome. Paediatr Anaesth 2002;12:461-4.

8. Johr M, Berger TM, Ruppen W, Schlegel C. Congenital laryngotracheo-oesophageal cleft: successful ventilation with the Laryngeal Mask Airway. Paediatr Anaesth 2003;13:68-71.

9. Leal-Pavey YR. Use of the LMA classic to secure the airway of a premature neonate with Smith-Lemli-Opitz syndrome: a case report. AANA J 2004;72:427-30.

10. Russell P, Chambers N, du Plessis J, Vijayasekeran S. Emergency use of a size 1 laryngeal mask airway in a ventilated neonate with an undiagnosed type IV laryngotracheo-oesophageal cleft. Paediatr Anaesth 2008;18:658-62.

11. Scheller B, Schalk R, Byhahn C, et al. Laryngeal tube suction II for difficult airway management in neonates and small infants. Resuscitation 2009;80:805-10.

12. Stocks RM, Egerman R, Thompson JW, Peery M. Airway management of the severely retrognathic child: use of the laryngeal mask airway. Ear Nose Throat J 2002;81:223-6.

13. Yao CT, Wang JN, Tai YT, Tsai TY, Wu JM. Successful management of a neonate with Pierre-Robin syndrome and severe upper airway obstruction by long term placement of a laryngeal mask airway. Resuscitation 2004;61:97-9.

14. Brunette DD, Fischer R. Intravascular access in pediatric cardiac arrest. Am J Emerg Med 1988;6:577-9.

15. Horton MA, Beamer C. Powered intraosseous insertion provides safe and effective vascular access for pediatric emergency patients. Pediatr Emerg Care 2008;24:347-50.

16. Banerjee S, Singhi SC, Singh S, Singh M. The intraosseous route is a suitable alternative to intravenous route for fluid resuscitation in severely dehydrated children. Indian Pediatr 1994;31:1511-20. 
17. Kanter RK, Zimmerman JJ, Strauss RH, Stoeckel KA. Pediatric emergency intravenous access: evaluation of a protocol. Am J Dis Child 1986;140:132-4.

18. Andropoulos DB, Soifer SJ, Schreiber MD. Plasma epinephrine concentrations after intraosseous and central venous injection during cardiopulmonary resuscitation in the lamb. J Pediatr 1990;116:312-5.

19. Johnson L, Kissoon N, Fiallos M, Abdelmoneim T, Murphy S. Use of intraosseous blood to assess blood chemistries and hemoglobin during cardiopulmonary resuscitation with drug infusions. Crit Care Med 1999;27:1147-52.

20. Johnston C. Endotracheal drug delivery. Pediatr Emerg Care 1992;8:94-7.

21. Kwon JW. High-flow nasal cannula oxygen therapy in children: a clinical review. Clin Exp Pediatr 2020;63:3-7.

22. Jasani MS, Nadkarni VM, Finkelstein MS, Mandell GA, Salzman SK, Norman ME. Effects of different techniques of endotracheal epinephrine administration in pediatric porcine hypoxic-hypercarbic cardiopulmonary arrest. Crit Care Med 1994; 22:1174-80

23. Sahin A, Meteroglu F, Eren $S$, Celik Y. Inhalation of foreign bodies in children: experience of 22 years. J Trauma Acute Care Surg 2013;74:658-63.

24. Raehl CL. Endotracheal drug therapy in cardiopulmonary resuscitation. Clin Pharm 1986;5:572-9.

25. Lubitz DS, Seidel JS, Chameides L, Luten RC, Zaritsky AL, Campbell FW. A rapid method for estimating weight and resuscitation drug dosages from length in the pediatric age group. Ann Emerg Med 1988;17:576-81.

26. Garland JS, Kishaba RG, Nelson DB, Losek JD, Sobocinski KA. A rapid and accurate method of estimating body weight. Am J Emerg Med 1986;4:390-3.

27. Young KD, Korotzer NC. Weight estimation methods in children: a systematic review. Ann Emerg Med 2016;68:441-51.

28. Jang HY, Shin SD, Kwak YH. Can the Broselow tape be used to estimate weight and endotracheal tube size in Korean children? Acad Emerg Med 2007;14:489-91.

29. So TY, Farrington E, Absher RK. Evaluation of the accuracy of different methods used to estimate weights in the pediatric population. Pediatrics 2009;123:e1045-51.

30. Hofer CK, Ganter M, Tucci M, Klaghofer R, Zollinger A. How reliable is length-based determination of body weight and tracheal tube size in the paediatric age group?: the Broselow tape reconsidered. Br J Anaesth 2002;88:283-5.

31. Niemann JT, Criley JM, Rosborough JP, Niskanen RA, Alferness C. Predictive indices of successful cardiac resuscitation after prolonged arrest and experimental cardiopulmonary resusci- tation. Ann Emerg Med 1985;14:521-8.

32. Sanders AB, Ewy GA, Taft TV. Prognostic and therapeutic importance of the aortic diastolic pressure in resuscitation from cardiac arrest. Crit Care Med 1984;12:871-3.

33. Andersen LW, Berg KM, Saindon BZ, et al. Time to epinephrine and survival after pediatric in-hospital cardiac arrest. JAMA 2015;314:802-10.

34. Jones $P$, Dauger $S$, Denjoy I, et al. The effect of atropine on rhythm and conduction disturbances during 322 critical care intubations. Pediatr Crit Care Med 2013;14:e289-97.

35. Valdes SO, Donoghue AJ, Hoyme DB, et al. Outcomes associated with amiodarone and lidocaine in the treatment of inhospital pediatric cardiac arrest with pulseless ventricular tachycardia or ventricular fibrillation. Resuscitation 2014;85: 381-6.

36. Dorian P, Cass D, Schwartz B, Cooper R, Gelaznikas R, Barr A. Amiodarone as compared with lidocaine for shock-resistant ventricular fibrillation. N Engl J Med 2002;346:884-90.

37. Quintard $H_{1}$ l'Her $E_{1}$ Pottecher J, et al. Experts' guidelines of intubation and extubation of the ICU patient of French Society of Anaesthesia and Intensive Care Medicine (SFAR) and French-speaking Intensive Care Society (SRLF): in collaboration with the pediatric Association of French-speaking Anaesthetists and Intensivists (ADARPEF), French-speaking Group of Intensive Care and Paediatric emergencies (GFRUP) and Intensive Care physiotherapy society (SKR). Ann Intensive Care 2019;9:13.

38. Fastle RK, Roback MG. Pediatric rapid sequence intubation: incidence of reflex bradycardia and effects of pretreatment with atropine. Pediatr Emerg Care 2004;20:651-5.

39. Jones $P$, Peters MJ, Pinto da Costa N, et al. Atropine for critical care intubation in a cohort of 264 children and reduced mortality unrelated to effects on bradycardia. PLoS One 2013; 8:e57478.

40. Beiser DG, Carr GE, Edelson DP, Peberdy MA, Hoek TL. Derangements in blood glucose following initial resuscitation from inhospital cardiac arrest: a report from the national registry of cardiopulmonary resuscitation. Resuscitation 2009;80:62430.

41. Rodriguez-Nunez A, Lopez-Herce J, del Castillo J, Bellon JM; Iberian-American Paediatric Cardiac Arrest Study Network RIBEPCI. Shockable rhythms and defibrillation during in-hospital pediatric cardiac arrest. Resuscitation 2014;85:387-91.

42. Gutgesell HP, Tacker WA, Geddes LA, Davis S, Lie JT, McNamara DG. Energy dose for ventricular defibrillation of children. Pediatrics 1976;58:898-901.

43. Meaney PA, Nadkarni VM, Atkins DL, et al. Effect of defibrilla- 
tion energy dose during in-hospital pediatric cardiac arrest. Pediatrics 2011;127:e16-23.

44. Torres-Andres F, Fink EL, Bell MJ, Sharma MS, Yablonsky EJ, Sanchez-de-Toledo J. Survival and long-term functional outcomes for children with cardiac arrest treated with extracorporeal cardiopulmonary resuscitation. Pediatr Crit Care Med 2018;19:451-8.

45. Pozzi $M$, Armoiry $X$, Achana $F$, et al. Extracorporeal life support for refractory cardiac arrest: a 10-year comparative analysis. Ann Thorac Surg 2019;107:809-16.

46. Park JH, Song KJ, Shin SD, Ro YS, Hong KJ. Time from arrest to extracorporeal cardiopulmonary resuscitation and survival after out-of-hospital cardiac arrest. Emerg Med Australas 2019;31:1073-81.

47. Meert KL, Guerguerian AM, Barbaro R, et al. Extracorporeal cardiopulmonary resuscitation: one-year survival and neurobehavioral outcome among infants and children with inhospital cardiac arrest. Crit Care Med 2019;47:393-402.

48. Lasa JJ, Rogers RS, Localio R, et al. Extracorporeal Cardiopulmonary Resuscitation (E-CPR) during pediatric in-hospital cardiopulmonary arrest is associated with improved survival to discharge: a report from the American Heart Association's Get With The Guidelines-Resuscitation (GWTG-R) registry. Circulation 2016;133:165-76.

49. Kramer P, Mommsen A, Miera O, Photiadis J, Berger F, Schmitt KR. Survival and mid-term neurologic outcome after extracorporeal cardiopulmonary resuscitation in children. Pediatr Crit Care Med 2020;21:e316-24.

50. Holmberg MJ, Geri G, Wiberg S, et al. Extracorporeal cardiopulmonary resuscitation for cardiac arrest: a systematic review. Resuscitation 2018;131:91-100.

51. Shin HJ, Song S, Park HK, Park YH. Results of extracorporeal cardiopulmonary resuscitation in children. Korean J Thorac Cardiovasc Surg 2016;49:151-6.

52. Shakoor A, Pedroso FE, Jacobs SE, et al. Extracorporeal Cardiopulmonary Resuscitation (ECPR) in infants and children: a single-center retrospective study. World J Pediatr Congenit Heart Surg 2019;10:582-9.
53. Conrad SA, Rycus PT. Extracorporeal membrane oxygenation for refractory cardiac arrest. Ann Card Anaesth 2017;20(Supplement):S4-10.

54. Bembea MM, Ng DK, Rizkalla N, et al. Outcomes After Extracorporeal Cardiopulmonary Resuscitation of Pediatric In-Hospital Cardiac Arrest: A Report From the Get With the Guidelines-Resuscitation and the Extracorporeal Life Support Organization Registries. Crit Care Med 2019;47:e278-85.

55. Tan BK. Extracorporeal membrane oxygenation in cardiac arrest. Singapore Med J 2017;58:446-8.

56. Lowry AW, Morales DL, Graves DE, et al. Characterization of extracorporeal membrane oxygenation for pediatric cardiac arrest in the United States: analysis of the kids' inpatient database. Pediatr Cardiol 2013;34:1422-30.

57. Ortmann L, Prodhan P, Gossett J, et al. Outcomes after in-hospital cardiac arrest in children with cardiac disease: a report from Get With the Guidelines-Resuscitation. Circulation 2011; 124:2329-37.

58. Odegard KC, Bergersen $L$, Thiagarajan $R$, et al. The frequency of cardiac arrests in patients with congenital heart disease undergoing cardiac catheterization. Anesth Analg 2014;118: 175-82.

59. de Mos N, van Litsenburg RR, McCrindle B, Bohn DJ, Parshuram CS. Pediatric in-intensive-care-unit cardiac arrest: incidence, survival, and predictive factors. Crit Care Med 2006;34:120915.

60. Pokorna M, Necas E, Kratochvil J, Skripsky R, Andrlik M, Franek 0 . A sudden increase in partial pressure end-tidal carbon dioxide $(P(E T) C O(2))$ at the moment of return of spontaneous circulation. J Emerg Med 2010;38:614-21.

61. Kleinman ME, Chameides L, Schexnayder SM, et al. Pediatric advanced life support: 2010 American Heart Association Guidelines for Cardiopulmonary Resuscitation and Emergency Cardiovascular Care. Pediatrics 2010;126:e1361-99.

62. Cantineau JP, Merckx P, Lambert $Y$, Sorkine $M$, Bertrand $C$, Duvaldestin P. Effect of epinephrine on end-tidal carbon dioxide pressure during prehospital cardiopulmonary resuscitation. Am J Emerg Med 1994;12:267-70. 